\title{
The Impact of an Online Cultural Simulation Activity on the Development of Speech Pathology Students' Cultural Empathy: A Pilot Study
}

Monica Lee ${ }^{a}$, Joanne Walters ${ }^{a}$, \& *Sally Hewat ${ }^{a}$

a: The University of Newcastle, Australia

\begin{abstract}
Despite the cultural and linguistic diversity of the Australian population, research suggests that speech pathologists do not feel confident when providing services to Cultural and Linguistic Diversity (CALD) clients and would benefit from further education and training in this area. Cultural empathy has been described as a precursor to cultural competence and previous research has demonstrated the positive impact on nursing students' cultural empathy toward CALD clients following an interactive cultural simulation experience. This study investigated the feasibility and effectiveness of an online cultural simulation activity in developing the cultural empathy of speech pathology students. Students completed an online cultural simulation in their own time, followed by a tutorial debrief. Both the simulation and debrief were considered to comprise the 'simulation activity'; which was a component of a first-year introduction to clinical practice course. Participants completed a pre-survey and post-survey, containing the Comprehensive Empathy Scale (CES) and demographic questions. Results were analysed using the Wilcoxon Signed Rank test and a Paired Sample t-test, and a power analysis was conducted to direct future studies. Ten students participated and improved an average of 11.3 on the CES $(p=0.14)$. A power analysis revealed that a minimum of 45 participants would be needed in future studies to observe any statistically significant results. The online application of the cultural simulation activity was feasible, and results indicate an improvement in cultural empathy, although the improvement did not reach statistical significance. Implications for future studies are discussed.
\end{abstract}

Keywords: cultural empathy; online; practice learning; simulation; speech pathology

\section{Introduction}

Australia is a multicultural country, rich in cultural and linguistic diversity (CALD). This term refers to groups that "differ according to religion and spirituality, racial backgrounds and ethnicity as well as language" (NHMRC, 2006). As globalisation has increased in recent years, so too has the CALD population within Australia: census data indicates that in 2011, 26\% of Australians were born overseas,

*Corresponding Author: Associate Professor Sally Hewat, School of Humanities \& Social Science, The University of Newcastle, Callaghan NSW 2308 Australia

Email: Sally.Hewat@newcastle.edu.au

Journal URL: https://publications.coventry.ac.uk/index.php/pblh

Lee, M., Walters, J., \& Hewat. S. (2020). The impact of an online cultural simulation activity on the development of speech pathology students' cultural empathy: A pilot study . International Journal of Practice-based Learning in Health and Social Care, 8(2), 16-28. DOI: 10.18552/ijpblhsc.v8i2.585.

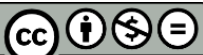

(C) BY ND (C) 2020 Monica Lee, Joanne Walters, \& *Sally Hewat. This Open Access article is distributed under the terms of the Creative Commons Attribution Attribution-Non-Commercial No Derivatives 4.0 International License (https://creativecommons.org/licenses/by-nc-nd/4.0/ ), which permits unrestricted noncommercial use, distribution, and reproduction in any medium, provided the original work is properly cited and is unaltered. 
and this group increased to 33.3\% in 2016 (Australian Bureau of Statistics [ABS], 2012; ABS, 2017). Furthermore, $19.3 \%$ of the Australian population spoke a language other than English in the home in 2011, while in 2016, this figure reached 21\% (ABS, 2012; ABS, 2017).

In contrast, Australian speech pathologists (SPs) are not a diverse population: Health Workforce Australia (2014) surveyed Australian SPs, and found that they were predominately Australian-born (82.9\%). Likewise, Williams \& McLeod (2012) found that only 9.3\% of SPs reported being 'proficient' in a language other than English. The survey also revealed a far smaller range of languages spoken among SPs when compared to the general population, with limited overlap between the languages spoken by the clinicians and their clients. The four most common languages spoken by surveyed SPs were French, Italian, German and Spanish, while the most common languages spoken by their clients were Vietnamese, Arabic, Cantonese and Mandarin (Williams \& McLeod, 2012).

This disparity indicates that Australian SPs are not representative of their client base with regards to culture and linguistic diversity, and that there is a low chance that bilingual SPs will speak the languages of their clients. Australian SPs are increasingly working overseas (Cocks \& Cruice, 2010; Pickering \& McAllister, 2000). As such, both in Australia and internationally, it is highly probable that SPs will service clients from outside their own cultural background.

Consideration of CALD clients is especially important in the health sector, as CALD populations have been noted to have poorer health outcomes than their mainstream Australian counterparts. This has been observed in chronic conditions such as diabetes and respiratory illness (Henderson et al., 2011) as well as cardiac and renal health (Juergens et al., 2016). Patients from CALD backgrounds are also less likely to seek health services (Henderson et al., 2011). These disparities are often attributed to language barriers and reduced health literacy (Komaric et al., 2012).

There is a clear necessity to address this disparity of health outcomes, but there is evidence suggesting that SPs feel that they are unable adequately to deliver services to CALD clients (Guiberson \& Atkins, 2010). This has been seen both in Australia, and internationally (Kritikos, 2003; Stow \& Dodd, 2003). This lack of confidence has been seen in various areas of speech pathology practice, including aphasia (Pang et al. 2015) and speech sound disorders (Verdon et al., 2015).

The necessity for SPs to work effectively with clients from various cultural and linguistically diverse backgrounds is evident, and therefore that SPs need to be culturally competent. Cultural competence involves developing self-awareness of one's own culture, learning about personal factors which may be culturally influenced, and then adapting personal and systemic clinical practice to ensure culturally appropriate service delivery for clients (Papadolpoulos et al., 1998; Wells, 2000). Further, Wells (2000) suggested that cultural competence involves balancing an awareness of both cultural and individual differences, in order to avoid stereotyping new clients based on cultural expectations. More recent views on cultural competence have diverged from viewing CALD clients as 'other', but rather recognise that every person has culture and, as such, every client embodies a cultural experience. These sentiments are reflected in speech pathology practice as well as healthcare more generally, as Verdon (2015) presented six key principles of culturally competent speech pathology practice, including "getting to know yourself", "forming relationships with families and communities" and "being flexible: one size does not fit all".

\section{Cultural empathy}

Empathy is considered a precursor to cultural competence (Sales et al., 2013), and is essential in creating open, trusting relationships with clients in order to achieve rapport and compliance, thereby improving therapy outcomes (Kim et al., 2004). Empathy has also been seen to reduce prejudice among healthcare professionals (Pettigrew \& Tropp, 2008).

Empathy is a complex and multifaceted phenomenon, comprising cognitive, emotional and behavioural components. It involves having a cognitive understanding of the emotions of others, allowing those emotions to resonate within oneself, and responding behaviourally by communicating this understanding to the other person (Batson, 2009; Scott, 2011; Wang et al., 2003). 
Like cultural competence, empathy is also a necessary attribute of practice in speech pathology, as outlined in the professional competencies for Australian SPs (Speech Pathology Australia, 2001). Ebert \& Kohnert (2010) conducted an analysis of clinician-related factors affecting therapy outcomes, finding that empathy and knowledge of CALD differences were key factors contributing to success in therapy.

Empathy relating specifically to CALD populations is known as cultural empathy (Wang et al., 2003). As with cultural competence more generally, there is evidence that cultural empathy requires development among healthcare professionals. Studies have shown that health professionals exhibit reduced empathy toward CALD populations (Johnson et al., 2004; Saha et al., 2008). For example, in a United States study, an investigation of empathy revealed a cultural bias which reduced pain treatment to AfricanAmerican patients, when compared with Caucasian patients; however, education in empathy was seen to reduce this bias and improve pain treatment (Drwecki et al., 2011). This need for development has also been observed in allied health professionals. Brown et al. (2010) found that the empathy of occupational therapy students is dependent on the medical condition of their client, with substance abuse eliciting the lowest level of empathy.

Speech Pathology Australia (2009) recommends embedding cultural competence coursework in the undergraduate curriculum, as cultural competence can be developed through education and training (Guiberson \& Atkins, 2010). In the last decade, the literature has focused increasingly on the nature of such cultural competence training; this has included CALD-specific subjects and the embedding of CALD-related lectures throughout university programs (Cheng et al., 2001; Lazewnik et al., 2010; Roseberry-McKibbin et al., 2005). In addition to lecture material, cultural competence can also be developed through intercultural clinical experience (Reichard et al., 2014), particularly when students engage in active reflection (Balandin et al., 2007; Verdon, 2015).

\section{Simulation}

Simulation is commonly used in medicine and nursing curricula to educate students in a wide variety of clinical skills (Kelly et al., 2016; Rogers et al., 2014), including empathy (Eymard et al., 2010; Jakes, 1999). Simulation-based learning has become increasingly common, as the under-supply of clinical placement sites remains a longstanding problem (McAllister, 2005; Speech Pathology Australia, 2018). As such, incorporating simulation at university has become an expedient method of providing experiences that students would previously have gained solely on clinical placement (Harder, 2010). This is particularly true with regard to intercultural experience, as not all domestic placement sites service a high number of CALD clients, and international placements are not practicable for all students (KentWilkinson et al., 2015). Therefore, given the evidence that intercultural experience and university coursework can improve cultural competence, it logically follows that simulation of such experience could serve as a feasible learning tool to improve students' cultural empathy.

The effect of simulation on cultural empathy has been explored in nursing. Everson et al. (2015) investigated the effect of a 3D cultural simulation activity on nursing students' cultural empathy. Participants experienced the simulation in the university clinic, by watching a 10 -minute video of a culturally unfamiliar medical scenario from the perspective of the patient, using simulation video glasses and accompanied by physical stimuli. For example, a researcher took participants' blood pressure while the patient in the video had their blood pressure taken. This was followed by a guided debrief. Results from this study indicated significant improvement in participants' cultural empathy following the 3D cultural simulation activity.

Simulation in speech pathology has been shown to be effective in developing clinical skills at undergraduate level (MacBean et al., 2013), as well as in further professional development such as tracheostomy training (Ward et al., 2014). However, to date, there is no research investigating simulation as a method of improving cultural empathy in speech pathology students. 


\begin{abstract}
Aims
The aim of the present study was to investigate the feasibility and effectiveness of an online adaptation of the cultural simulation activity presented by Levett-Jones (2015) and Everson et al. (2015), in improving the cultural empathy of speech pathology students. A further aim was to complete a power analysis to determine the required sample size for future studies.
\end{abstract}

\title{
Ethical clearance
}

The university ethics committee granted ethical approval for the study, reference number H-2016-0253. Participants provided informed consent to participate in the study.

\section{Cultural simulation activity}

A cultural simulation learning activity, based on the work of Everson et al. (2015), was developed and embedded in a first-year introduction to clinical practice course. The learning activity followed a 'flipped classroom' format, wherein students independently watched an online video of an intercultural experience, then attended a tutorial to debrief and discuss the video. Both the video and debrief comprised the cultural simulation activity.

The video experience was adapted from Everson et al. (2015). This involved the students watching the same 10-minute video as presented by Everson and colleagues; however, in the present study, all students enrolled in the course independently watched the video online rather than through simulation video glasses at the university and without the physical stimuli. They were instructed to lie down in a dark room, as if in a hospital bed. They were instructed to imagine that the scene was real; that they had been travelling through a foreign country before becoming acutely unwell and being admitted to hospital. A detailed instruction sheet was provided on the students' online learning platform. Additional support was also provided by lecturers and tutors via an online discussion forum.

The video was presented from the perspective of the patient and portrayed a hospitalisation experience in a non-Anglo-Saxon culture, showing a crowded hospital room with non-English-speaking hospital staff. Visual and auditory disturbances were included to simulate medical symptoms. The video was available to the students online for one week.

In the following week, students attended a tutorial (a tutorial group comprising a maximum of 20 students) in which they debriefed their experience of viewing the video. The discussion was guided by the course tutor using questions which were also adapted from Everson et al. (2015). The questions initially centred around feelings arising from the video (for example, If the simulation was a real situation how might you, as the patient, have been feeling as the scenario unfolded and why?). Students were also provided with information about health outcomes for CALD populations and engaged in discussions regarding the role of speech pathologists in improving these outcomes.

\section{Comprehensive Empathy Scale}

The cultural empathy of students was measured using the Comprehensive Empathy Scale (CES) (LevettJones, 2015). The CES has a Cronbach's alpha of 0.96, indicating strong internal consistency (LevettJones, 2015; Tavakol \& Dennick, 2011). Further, it measures "state empathy" not "trait empathy". That is, the scale measures a person's empathic response at a particular point in time, rather than the extent to which empathy is reflected in their personality. As such, it is an appropriate measure of short-term change in empathy (Levett-Jones, 2015).

The CES involves a pre-test and a post-test, each containing written accounts of hospital experiences from the point of view of different CALD patients. These stories are true, but with details changed to protect confidentiality. Both stories are accompanied by an image of a CALD patient who appeared distressed. 
In the CES pre- and post-tests, participants were asked a total of 39 questions. Questions 1-14 asked participants to rate from 1 ("not at all") to 5 ("very much") the extent to which they experienced the given feelings while reading the story. For example, these feelings included "compassionate, distressed, afraid". Questions 15-39 asked participants to indicate the extent to which a series of statements were true for them, from 1 ("completely untrue") to 5 ("completely true"). The statements included "I found that the scenario affected my mood". The CES was scored through addition of participant responses of 15 for each of the 39 questions, yielding a total possible score of 39-195. Higher scores on this scale indicated higher empathy.

As well as yielding an overall empathy score, the CES was subcategorised into six subtests to reflect the multifaceted nature of empathy, based on Batson's eight conceptualisations of empathy (Batson, 2009; Levett-Jones, 2015).

\section{Data collection procedures}

Students who agreed to participate in the research were required to complete pre- and post-surveys, before and after the cultural simulation activity. Participants independently completed the surveys online through SurveyMonkey®, available via the students’ online learning platform.

The pre-survey was completed in the week before the tutorial, prior to the video simulation becoming available. The pre-survey included demographic questions to identify personal factors which may influence the development of cultural empathy as well as the CES pre-test. The post-survey containing the CES post-test was completed in the week following the tutorial (that is, within 3 weeks of completing the pre-survey). For both surveys, participants were asked to provide a unique identifying code in order to match pre-survey and post-survey responses anonymously.

\section{Participants}

This study recruited first-year undergraduate speech pathology students at a regional Australian university. To be eligible for the study, participants were required to be enrolled in 'SPTH1080 Introduction to Clinical Practice'. This is the first opportunity for students enrolled in the speech pathology program to learn about CALD and take part in a simulated learning activity. SP students were invited to participate in the research via email, the online learning management system BlackBoard, and in person during a lecture. There were no exclusion criteria: all students enrolled in the specific course SPTH1080 were able to participate.

\section{Analysis}

Demographic data were analysed using descriptive analysis (e.g. frequency, percentages) (Campbell 2016). The feasibility of the online adaptation of the cultural simulation activity was determined by examining the percentage of students on the course who engaged with both the online video and tutorial debrief. This data was collected through Blackboard ${ }^{\odot}$, and the attendance recorded at the tutorials.

The overall difference between the pre-survey and post-survey CES scores was analysed using a Paired Sample t-test, an assumption of which was that the data set was normally distributed, and a Wilcoxon Signed Rank Test, which does not make this assumption (McCrum-Gardner, 2008). Both analyses were completed, as normality of the data could not be determined given the small sample size. Two-tailed, rather than one-tailed tests were conducted, as it was determined that there was insufficient rationale for a one-tailed test, as a negative trend on the CES could reflect valid and meaningful results (Eng, 2003). Further, a two-tailed approach was preferred as it encompassed the more conservative approach (Eng, 2003).

Differing empathy scores in relation to CES subscales and demographic factors of participants were not statistically analysed, as the sample size was determined to be too small to reveal any significance in the results (MacCallum et al., 1999). A power analysis was conducted to determine the required sample size 
needed for future studies to detect a statistically significant change on the CES, should it occur (Cohen, 1992).

\section{Results}

Of a possible 92 students in the course, 17 completed the pre-survey and 15 completed the post-survey. Participants who only completed one of the two surveys were excluded. A total of 11 participants completed both surveys and are reported in this paper. Participant 11 did not complete the entire simulation activity (online video plus tutorial debrief) and was therefore excluded from the statistical analysis evaluating the change in cultural empathy following the simulation activity.

Demographic information for the participants is provided in Table 1. Participants were aged 18-40 years ( median $=20)$. Most participants were born in Australia $(n=9)$ and three participants had at least one parent born overseas. One participant identified as Aboriginal/Torres Strait Islander. Some participants had lived in another country for more than 12 months $(n=2)$ and spoke at least one other language to a proficient level. All participants reported speaking only English at home. Six participants had completed the only clinical placement available to first-year students, a clinical observation day.

Table 1: Demographic information of participants

\begin{tabular}{|c|c|c|c|c|c|c|}
\hline Participant & Age & $\begin{array}{l}\text { Country } \\
\text { of birth }\end{array}$ & $\begin{array}{l}\text { At least } \\
\text { one } \\
\text { parent } \\
\text { born } \\
\text { overseas }\end{array}$ & $\begin{array}{l}\text { Language(s) } \\
\text { other than } \\
\text { English } \\
\text { spoken }\end{array}$ & $\begin{array}{l}\text { Countries } \\
\text { lived in } \\
\text { other than } \\
\text { Australia }\end{array}$ & $\begin{array}{l}\text { Completed } \\
\text { clinical } \\
\text { observation } \\
\text { day }\end{array}$ \\
\hline 1 & 19 & Australia & Yes & None & None & Yes \\
\hline 2 & 27 & Australia & No & $\begin{array}{l}\text { Italian } \\
\text { French }\end{array}$ & Italy & No \\
\hline 3 & 19 & Australia & No & None & None & Yes \\
\hline 4 & 20 & Singapore & Yes & Mandarin & $\begin{array}{l}\text { Singapore, } \\
\text { United } \\
\text { Kingdom }\end{array}$ & Yes \\
\hline 5 & 20 & Australia & No & None & None & Yes \\
\hline 6 & 40 & Australia & No & None & None & Yes \\
\hline 7 & 23 & Australia & No & None & None & No \\
\hline 8 & 40 & Australia & No & None & None & Yes \\
\hline 9 & 18 & Australia & Yes & None & None & No \\
\hline 10 & 20 & Australia & No & None & None & No \\
\hline $11^{*}$ & 20 & $\begin{array}{l}\text { New } \\
\text { Zealand }\end{array}$ & Yes & $\begin{array}{l}\text { Italian } \\
\text { Maori }\end{array}$ & $\begin{array}{l}\text { New } \\
\text { Zealand }\end{array}$ & Yes \\
\hline
\end{tabular}

*Participant 11 did not complete all components of the online cultural simulation activity

\section{Feasibility}

Although only 11 students participated in the study, additional data gathered from analytics in Blackboard indicated that the online cultural simulation video was accessed by 65 students out of 92 enrolled in the course $(70.7 \%)$ with 80 students attending the tutorial debrief $(87.0 \%)$.

\section{Effectiveness}


Individual and average CES scores are outlined in Table 2. Participants improved by an average of 11.3 points on the CES. This difference approached, but did not reach, statistical significance in the Paired Sample t-test $(p=0.17)$ or the Wilcoxon Signed Rank Test $(p=0.14)$. Participant 11 , who did not complete the cultural simulation activity, scored 177 on the pre-survey and 193 on the post-survey, an improvement of 16 points on the CES. Their result was not included in the overall analysis of average participant scores as they did not complete the simulation activity.

Table 2: Individual and average participant scores on the Comprehensive Empathy Scale (CES)

\begin{tabular}{|l|l|l|l|}
\hline Participant & Pre-score & Post-score & Difference \\
\hline 1 & 138 & 139 & 1 \\
\hline 2 & 160 & 123 & -37 \\
\hline 3 & 169 & 173 & 4 \\
\hline 4 & 151 & 181 & 30 \\
\hline 5 & 126 & 171 & 45 \\
\hline 6 & 156 & 142 & -14 \\
\hline 7 & 113 & 141 & 28 \\
\hline 8 & 102 & 116 & 14 \\
\hline 9 & 163 & 178 & 15 \\
\hline 10 & 116 & 143 & 27 \\
\hline AVERAGE: & $\mathbf{1 3 9 . 4}$ & $\mathbf{1 5 0 . 7}$ & $\mathbf{1 1 . 3}$ \\
\hline $11^{*}$ & 177 & 193 & 16 \\
\hline
\end{tabular}

* Data was not included in analysis

\section{Power analysis}

Although there is evidence of improvement in empathy following the simulation activity, the small number of participants rendered statistical significance unlikely. As such, a power analysis was performed to determine the minimum number of participants needed in future studies to reveal whether the simulation activity results in a statistically significant change in participants' cultural empathy. Power analyses require calculation of four variables to determine the required number of participants: significance level $(\alpha)$, power $(\beta)$, variability, and effect size.

Convention was followed in determining the values for the significance level and power, $\alpha=0.05$, and $\beta$ $=0.8$, as this is viewed as an acceptable balance of the risks of a Type I error (false positive claim) as compared to a Type II error (false negative claim) (Cohen, 1992; Eng, 2003).

Variability was estimated based on the standard deviation (SD) of the difference in pre- and post-CES scores in the pilot data (Table 2). The SD observed from our pilot data was 23.95389. The higher the variance of results in a population, the more participants are needed to produce statistically significant results, if they exist (Kraemer \& Thiemann, 1987). A confidence interval (CI) estimate of the population SD was performed to reduce the risk of underestimating the SD. Power analyses were conducted using an SD of 23.95389 and 35. A variance of 35 was chosen in addition as it was the upper limit of an $80 \%$ CI analysis and that was deemed realistic and sufficient.

The data from this pilot study provided an estimated effect size, that is, the difference in scores on the CES resulting from the cultural simulation activity. An effect size of 10 was taken to be the most likely. This was based on two methods of hypothesising effect size. The first was data from this pilot study 
Figure 1: Interaction between power, sample size and effect size on the CES, assuming a standard deviation of 23.95389

Paired t-test: Power vs Sample Size

(for a range of possible Values of the true mean difference in Pre and Post CES Scores)

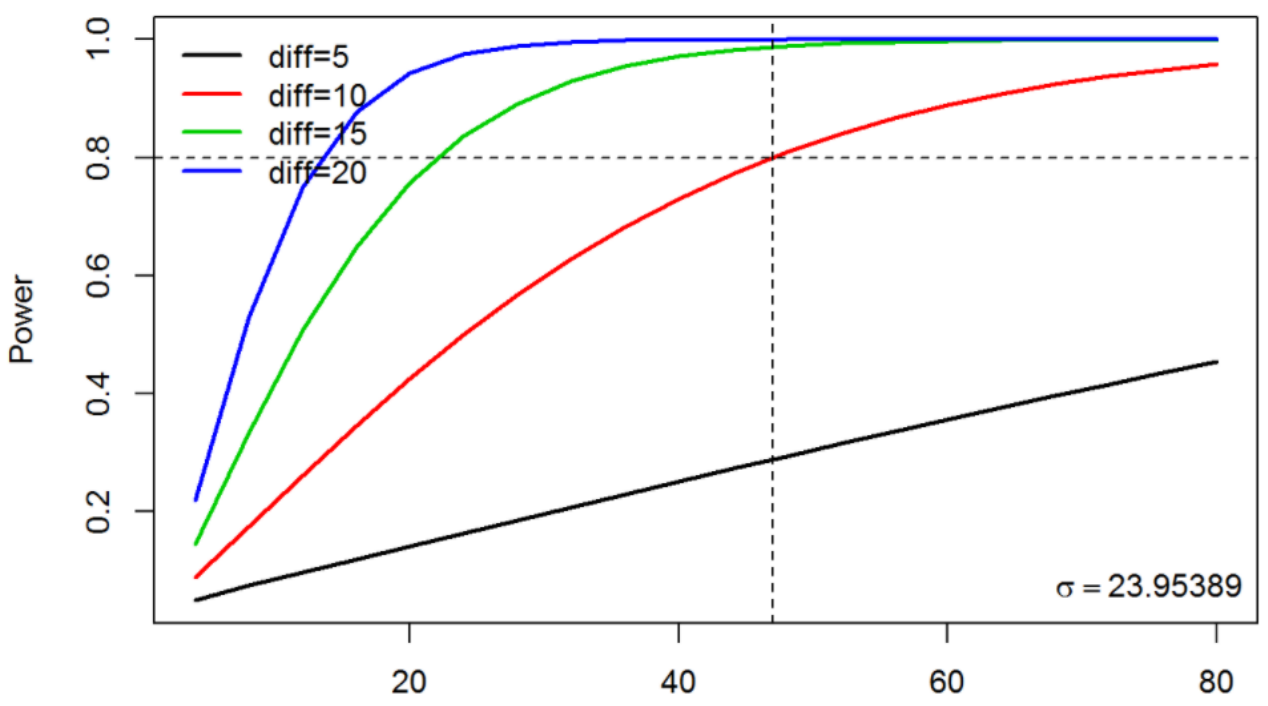

Sample Size

Figure 2: Interaction between power, sample size and effect size on the CES, assuming a standard deviation of 35

Paired t-test: Power vs Sample Size

(for a range of possible Values of the true mean difference in Pre and Post CES Scores)

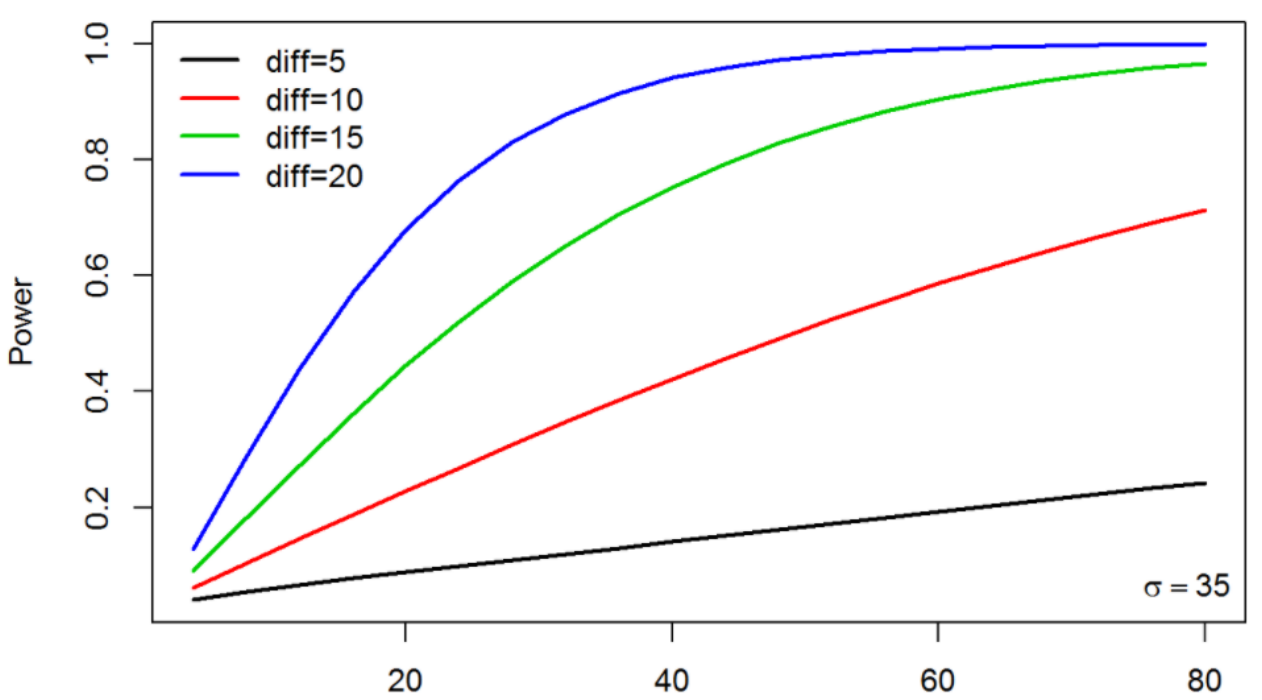

Sample Size 
which was 11.3, and the second was taking half of the SD, in this case 11.5 (Bausell \& Li, 2002; Cohen, 1992). This was rounded down to 10 as a conservative measure, as smaller effect sizes require larger sample sizes (Cohen, 1992)

Based on a calculation of the above variables, including SD=23.95389, approximately 45 participants would be ideal to observe statistically significant results in both a Paired Sample t-test (Figure 1) and Wilcoxon Signed Rank Test. Alternatively if the SD is taken to be 35, more than 80 participants would be needed (Figure 2) in future studies.

\section{Discussion}

The results from this preliminary study suggest an improvement in the cultural empathy of speech pathology students following an online cultural simulation activity. While the results did not reach our assigned level of statistical significance, this would have been an unlikely outcome given the small sample size (Crawley, 2015). The results are sufficiently promising to warrant further studies and have provided valuable information regarding power analysis and experimental design for such studies.

Further, the online adaptation of the cultural simulation activity was found to be feasible due to the high percentage of student engagement. It should also be noted that the adaptation of the cultural simulation activity involved only five hours of face-to-face teaching time, far less than the 3D experience outlined by Everson et al. (2015). In terms of equipment, the adapted simulation only involved a computer, which is owned by most students, or freely available in the university library.

Results revealed several interesting trends in the CES data. For example, some participant scores decreased between the pre-survey and post-survey. This may have been due to overconfidence in the presurvey, or increased awareness of the limitations of their cultural understanding in the post-survey.

Another point of interest was the performance of Participant 11, who completed both surveys but did not complete the online cultural simulation activity. Participant 11 scored an above average improvement of 16 on the CES and achieved a post-survey score of 193; the highest of all participants. It is possible that personal demographic factors could explain these results. For example, Participant 11 was born overseas, lived overseas for a significant period of time, spoke two languages other than English, had previous personal experience with CALD populations and completed a clinical observation day during the course of the study. Experience with other cultures has been seen to develop cultural competence (Gibson \& Zhong, 2005), so it is possible that the culmination of these personal factors influenced the high CES results. The clinical observation day could account for the change, as clinical experience can also develop cultural competence (Howells et al., 2016; Ng et al., 2012).

While it is possible that the personal circumstances of Participant 11 influenced their CES results, the variance in results from other participants who share these factors does not seem to support this conjecture. Ultimately the impact of personal demographic factors and clinical experience cannot be observed in such small data sets. Statistical significance cannot be determined, and further study involving a control group must be conducted to differentiate between the effects of the cultural simulation and of potential confounding factors (Campbell et al., 2000; Stephenson \& Imrie, 1998).

It is also possible that the validity of the CES was compromised by the reactive interaction effect of testing (Campbell \& Stanley, 2015). That is, completing the CES itself may affect participant perceptions of the cultural simulation activity, as they may be more open to the experiences in the simulation, having recently been exposed to a similar scenario. Further, it is possible that the CES may directly affect a person's cultural empathy. Given that the survey portrays a hospitalisation experience from the perspective of a CALD patient, the CES could be considered similar to the cultural simulation activity. However, it should be noted that the cultural simulation activity includes the tutorial debrief, which involves guided reflection, and students are presented with information regarding CALD healthcare in Australia. These aspects of the overall simulation are key, as the development of cultural competence requires reflection on one's own cultural beliefs and on education about CALD in healthcare 
(Papadopoulos et al., 1998; Wells, 2000). Nevertheless, the validity of the CES should be examined in future studies for a better understanding of the impact of any reactive interaction effect of testing.

\section{Conclusions}

The current study was a pilot project designed to determine the feasibility of an online cultural simulation activity and gain preliminary findings regarding its effectiveness. The findings from this study should therefore be interpreted cautiously, due to the small sample size, the lack of a control group, and to the involvement of participants from only one university. Investigating the long-term maintenance of treatment effects was also beyond the scope of this study. Furthermore, given the independent online nature of the cultural simulation activity, it is uncertain whether students completed and viewed the online video simulation as instructed.

Further studies should involve a minimum of 45-80 participants in order to observe any existing statistically significant effect of the online cultural simulation activity. Future studies should also include statistical consideration of personal demographic factors which may potentially affect the development of cultural empathy (Reichard et al., 2014). The inclusion of a control group is also recommended, as this would make it possible to distinguish between the treatment effect and other factors which may be influencing the results (Campbell et al., 2000; Stephenson \& Imrie, 1998).

While the results did not reach statistical significance, they do indicate that the implementation of an online cultural simulation activity may be a feasible means of developing the cultural empathy of speech pathology students.

\section{Acknowledgements}

The authors would like to acknowledge Professor Tracy Levett-Jones, Conjoint Professor at the University of Newcastle, and her colleagues for their work in the development of the cultural empathy toolkit used in this study..

\section{Ethical approval}

The University of Newcastle Human Research Ethics Committee: Approval No. H-2016-0253.

\section{ORCID}

Monica Lee $\quad$ https://orcid.org/0000-0003-0499-9918

Joanne Walters https://orcid.org/0000-0002-3051-1122

Sally Hewat $\quad$ https://orcid.org/0000-0002-7043-7086

\section{References}

ABS (Australian Bureau of Statistics). (2012). Cultural Diversity in Australia (No. 2071.0). http://www.abs.gov.au/ausstats/

ABS (Australian Bureau of Statistics). (2017). Census of Population and Housing: Reflecting Australia - Stories from the Census, 2016 (No. 2071.0). http://www.abs.gov.au/ausstats

Balandin, S., Lincoln, M., Sen, R., Wilkins, D. P., \& Trembath, D. (2007). Twelve tips for effective international clinical placements. Medical Teacher, 29(9-10), 872-877. https://doi.org/10.1080/01421590701784364

Batson, C. D. (2009) These things called empathy: Eight related but distinct phenomena. In J. Decety \& W. Ickes (Eds.), The social neuroscience of empathy (pp. 3-16). MIT Press. https://doi.org/10.7551/mitpress/9780262012973.003.0002

Bausell, R. B., \& Li, Y. F. (2002). Power analysis for experimental research: A practical guide for the biological, medical and social sciences. Cambridge University Press. https://doi.org/10.1017/CBO9780511541933 
Brown, T., Williams, B., Boyle, M., Molloy, A., McKenna, L., Molloy, L., \& Lewis, B. (2010). Levels of empathy in undergraduate occupational therapy students. Occupational Therapy International, 17(3), 135-141. https://doi.org/10.1002/oti.297

Campbell, D. T., \& Stanley, J. C. (2015). Experimental and quasi-experimental designs for research. Handbook of research on teaching. Ravenio Books.

Campbell, M. (2016). Getting to grips with statistics: Understanding variables. British Journal of Midwifery, 24(10), 738-741. https://doi.org/10.12968/bjom.2016.24.10.738

Campbell, M., Fitzpatrick, R., Haines, A., Kinmonth, A. L., Sandercock, P., Spiegelhalter, D., \& Tyrer, P. (2000). Framework for design and evaluation of complex interventions to improve health. British Medical Journal, 321(7262), 694-696. https://doi.org/10.1136/bmj.321.7262.694

Cheng, L., Battle, D., Murdoch, B., \& Martin, D. (2001). Educating speech-language pathologists for a multicultural world. Folia Phoniatrica et Logopaedica, 53(3), 121-127. https://doi.org/10.1159/000052666

Cocks, N., \& Cruice, M. (2010). The experiences and perspectives of overseas trained speech and language therapists working in the United Kingdom. International Journal of Speech-Language Pathology, 12(3), 271-282. https://doi.org/10.3109/17549500903215318

Cohen, J. (1992). Statistical power analysis. Current Directions in Psychological Science, 1(3), 98-101. https://doi.org/10.1111/1467-8721.ep10768783

Crawley, M. (2015) Statistics: An introduction using $R$ (2nd ed.). Wiley.

Drwecki, B. B., Moore, C. F., Ward, S. E., \& Prkachin, K. M. (2011). Reducing racial disparities in pain treatment: The role of empathy and perspective-taking. Pain, 152(5), 1001-1006. https://doi.org/10.1016/i.pain.2010.12.005

Ebert, K. D., \& Kohnert, K. (2010). Common factors in speech-language treatment: An exploratory study of effective clinicians. Journal of Communication Disorders, 43(2), 133-147. https://doi.org/10.1016/j.jcomdis.2009.12.002

Eng, J. (2003). Sample size estimation: How many individuals should be studied? Radiology, 227(2), 309-313. https://doi.org/10.1148/radiol.2272012051

Everson, N., Levett-Jones, T., Lapkin, S., Pitt, V., Riet, P., Rossiter, R., Jones, D., Gilligan, C., \& Courtney-Pratt, H. (2015). Measuring the impact of a 3D simulation experience on nursing students' cultural empathy using a modified version of the Kiersma-Chen Empathy Scale. Journal of Clinical Nursing, 24(19-20), 2849-2858. https://doi.org/10.1111/jocn.12893

Eymard, A. S., Crawford, B. D., Keller, T. M., \& Campbell, S. L. (2010). "Take a walk in my shoes": Nursing students take a walk in older adults' shoes to increase knowledge and empathy. Geriatric Nursing, 31(2), 137-141. https://doi.org/10.1016/j.gerinurse.2010.02.008

Gibson, D., \& Zhong, M. (2005). Intercultural communication competence in the healthcare context. International Journal of Intercultural Relations, 29(5), 621-634. https://doi.org/10.1016/j.ijintrel.2005.07.008

Guiberson, M., \& Atkins, J. (2010). Speech-language pathologists' preparation, practices, and perspectives on serving culturally and linguistically diverse children. Communication Disorders Quarterly, 33(3), 169-180. https://doi.org/10.1177/1525740110384132

Harder, B. N. (2010). Use of simulation in teaching and learning in health sciences: A systematic review. Journal of Nursing Education, 49(1), 23-28. https://doi.org/10.3928/01484834-20090828-08

Health Workforce Australia. (2014). Speech pathologists in focus. Australia's Health Workforce Series. http://www.hwa.gov.au/publication/speech-pathologistsfocus-0

Henderson, S., Kendall, E., \& See, L. (2011). The effectiveness of culturally appropriate interventions to manage or prevent chronic disease in culturally and linguistically diverse communities: A systematic literature review. Health and Social Care in the Community, 19(3), 225-249. https://doi.org/10.1111/j.13652524.2010.00972.x

Howells, S., Barton, G., \& Westerveld, M. (2016). Exploring the development of cultural awareness amongst postgraduate speech-language pathology students. International Journal of Speech-Language Pathology, 18(3), 1-16. https://doi.org/10.3109/17549507.2016.1154982

Jakes, M. A. (1999). Trained empathy: A simulated patient experience (Doctoral dissertation). http://trace.tennessee.edu/utk_chanhonoproj/

Johnson, R. L., Saha, S., Arbelaez, J. J., Beach, M. C., \& Cooper, L. A. (2004). Racial and ethnic differences in patient perceptions of bias and cultural competence in health care. Journal of General Internal Medicine, 19(2), 101-110. https://doi.org/10.1111/j.1525-1497.2004.30262.x

Juergens, C. P., Dabin, B., French, J. K., Kritharides, L., Hyun, K., Kilian, J., Chew, D., \& Brieger, D. (2016). English as a second language and outcomes of patients presenting with acute coronary syndromes: Results from the CONCORDANCE registry. Medical Journal of Australia, 204(6), 239. https://doi.org/10.5694/mja15.00812

Kelly, M. A., Hopwood, N., Rooney, D., \& Boud, D. (2016). Enhancing students' learning through simulation: Dealing with diverse, large cohorts. Clinical Simulation in Nursing, 12(5), 171-176. https://doi.org/10.1016/i.ecns.2016.01.010 
Kent-Wilkinson, A., Leurer, M.D., Luimes, J., Ferguson, L., \& Murray, L. (2015). Studying abroad: Exploring factors influencing nursing students' decisions to apply for clinical placements in international settings. Nurse Education Today, 35(8), 941-947. https://doi.org/10.1016/j.nedt.2015.03.012

Kim, S. S., Kaplowitz, S., \& Johnston, M. V. (2004). The effects of physician empathy on patient satisfaction and compliance. Evaluation and the Health Professions, 27(3), 237-251. https://doi.org/10.1177/0163278704267037

Komaric, N., Bedford, S., \& van Driel, M. (2012). Two sides of the coin: Patient and provider perceptions of health care delivery to patients from culturally and linguistically diverse backgrounds. BMC Health Services Research, 12(1), 322. https://doi.org/10.1186/1472-6963-12-322

Kraemer, H. C., \& Thiemann, S. (1987). How many subjects? Statistical power analysis in research (1st ed.). Sage.

Kritikos, E. P. (2003). Speech-language pathologists' beliefs about language assessment of bilingual/bicultural individuals. American Journal of Speech-Language Pathology, 12(1), 73-91. https://doi.org/10.1044/10580360(2003/054)

Lazewnik, R., Creaghead, N., Combs, S., \& Raisor-Becker, L. (2010). Perspectives on preparing graduate students to provide services to diverse populations in schools. SIG 16: Perspectives on School-Based Issues, 11(2), 3339. https://doi.org/10.1044/sbi11.2.33

Levett-Jones, T. (2015). Cultural Empathy Toolkit. Newcastle, NSW: The University of Newcastle.

MacBean, N., Theodoros, D., Davidson, B., \& Hill, A. E. (2013). Simulated learning environments in speechlanguage pathology: An Australian response. International Journal of Speech-Language Pathology, 15(3), 345-357. https://doi.org/10.3109/17549507.2013.779024

MacCallum, R. C., Widaman, K. F., Zhang, S., \& Hong, S. (1999). Sample size in factor analysis. Psychological Methods, 4(1), 84-99. https://doi.org/10.1037/1082-989X.4.1.84

McAllister, L. (2005). Issues and innovations in clinical education. Advances in Speech-Language Pathology, 7(3), 138-148. https://doi.org/10.1080/14417040500181239

McCrum-Gardner, E. (2008). Which is the correct statistical test to use? British Journal of Oral and Maxillofacial Surgery, 46(1), 38-41. https://doi.org/10.1016/j.bjoms.2007.09.002

Ng, P., Goddard, T., Gribble, N., \& Pickard, C. (2012). International placements increase the cultural sensitivity and competency of allied health students: A quantitative and qualitative study. Journal of Physical Therapy Education, 26(1), 61-68. https://doi.org/10.1097/00001416-201210000-00011

NHMRC (National Health and Medical Research Council). (2006). Cultural competency in health: A guide for policy, partnerships and participation. https://www.nhmrc.gov.au/guidelines-publications/hp19-hp26

Pang, S., Mok, Z., \& Rose, M. (2015). Time for change: Results of a national survey of SLP practice in CALD aphasia rehabilitation. Journal of Clinical Practice in Speech-Language Pathology, 17(1), 20-26. https://cld.bz/bookdata/3I0D8sa/basic-html/page-22.html\#

Papadopoulos, I., Tilki, M., \& Taylor, G. (1998). Transcultural care: A guide for healthcare professionals. Quay Books.

Pettigrew, T. F., \& Tropp, L. R. (2008). How does intergroup contact reduce prejudice? Meta-analytic tests of three mediators. European Journal of Social Psychology, 38(6), 922-934. https://doi.org/10.1002/ejsp.504

Pickering, M., \& McAllister, L. (2000). A conceptual framework for linking and guiding domestic cross-cultural and international practice in speech-language pathology. Advances in Speech Language Pathology, 2(2), 93106. https://doi.org/10.3109/14417040008996794

Reichard, R., Serrano, S., Condren, M., Wilder, N., Dollwet, M., \& Wang, W. (2014). Engagement in cultural trigger events in the development of cultural competence. Academy of Management Learning \& Education, 14(4), 461-481. https://doi.org/10.5465/amle.2013.0043

Rogers, G. D., McConnell, H. W., De Rooy, N. J., Ellem, F., \& Lombard, M. (2014). A randomised controlled trial of extended immersion in multi-method continuing simulation to prepare senior medical students for practice as junior doctors. BMC Medical Education, 14(1), 90-100. https://doi.org/10.1186/1472-6920-14-90

Roseberry-McKibbin, C., Brice, A., \& O'Hanlon, L. (2005). Serving English language learners in public school settings: A national survey. Language, Speech and Hearing Services in Schools, 36(1), 48-61. https://doi.org/10.1044/0161-1461(2005/005)

Saha, S., Beach, M. C., \& Cooper, L. A. (2008). Patient centeredness, cultural competence and healthcare quality. Journal of the National Medical Association, 100(11), 1275-1285. https://doi.org/10.1016/S00279684(15)31505-4

Sales, I., Jonkman, L., Connor, S., \& Hall, D. (2013). A comparison of educational interventions to enhance cultural competency in pharmacy students. American Journal of Pharmaceutical Education, 77(4), 76. https://doi.org/10.5688/ajpe77476

Scott, H. (2011). Empathy in healthcare settings (Doctoral dissertation). http://research.gold.ac.uk/id/eprint/6704

Speech Pathology Australia (2001). Competency-based occupational standards (CBOS) for speech pathologists. SPA Limited.

Speech Pathology Australia. (2009). Working in a culturally and linguistically diverse society. SPA Limited.

Speech Pathology Australia. (2018) Clinical Education in Australia: Building a Profession for the Future. SPA Limited. 
Stephenson, J., \& Imrie, J. (1998). Why do we need randomised controlled trials to assess behavioural interventions? British Medical Journal, 316(7131), 611-613. https://doi.org/10.1136/bmj.316.7131.611

Stow, C., \& Dodd, B. (2003). Providing an equitable service to bilingual children in the UK: A review. International Journal of Language \& Communication Disorders, 38(4), 351-377. https://doi.org/10.1080/1368282031000156888

Tavakol, M., \& Dennick, R. (2011). Making sense of Cronbach's alpha. International Journal of Medical Education, 2(1), 53-55. https://doi.org/10.5116/ijme.4dfb.8dfd

Verdon, S. (2015). Enhancing practice with culturally and linguistically diverse families: 6 key lessons from the field. Journal of Clinical Practice in Speech-Language Pathology, 17(1), 2-6.

Verdon, S., McLeod, S., \& Wong, S. (2015). Reconceptualizing practice with multilingual children with speech sound disorders: People, practicalities and policy. International Journal of Language \& Communication Disorders, 50(1), 48-62. https://doi.org/10.1111/1460-6984.12112

Wang, Y. W., Davidson, M. M., Yakushko, O. F., Savoy, H. B., Tan, J. A., \& Bleier, J. K. (2003). The scale of ethnocultural empathy: Development, validation, and reliability. Journal of Counselling Psychology, 50(2), 221-234. https://doi.org/10.1037/0022-0167.50.2.221

Ward, E. C., Baker, S. C., Wall, L. R., Duggan, B. L., Hancock, K. L., Bassett, L. V., \& Hyde, T. J. (2014). Can human mannequin-based simulation provide a feasible and clinically acceptable method for training tracheostomy management skills for speech-language pathologists? American Journal of Speech-Language Pathology, 23(3), 421-436. https://doi.org/10.1044/2014_AJSLP-13-0050

Wells, M. (2000). Beyond cultural competence: A model for individual and institutional cultural development. Journal of Community Health Nursing, 17(4), 189-199. https://doi.org/10.1207/S15327655JCHN1704_1

Williams, C., \& McLeod, S. (2012). Speech-language pathologists' assessment and intervention practices with multilingual children. International Journal of Speech-Language Pathology, 14(3), 292-305. https://doi.org/10.3109/17549507.2011.636071 